\title{
Descrição da larva de Scinax similis (Cochran) com notas comparativas sobre o grupo "ruber" no sudeste do Brasil (Amphibia, Anura, Hylidae)
}

\author{
Ana C.R. Alves ${ }^{1,2}$ \\ Sergio Potsch de Carvalho e Silva ${ }^{1}$
}

\begin{abstract}
Description of the larva of Scinax similis (Cochran) with comparative notes on the Scinax ruber group in Southeastern Brazil (Amphibia, Anura, Hylidae). The larva of Scinax similis (Cochran, 1952) is described from Ilha do Fundão (Rio de Janeiro, Rio de Janeiro State, Brazil). Comparisons with other species of the group that occur in Southeastern Brazil are added. The larva of S. similis can be distinguished by the following diagnostic features: (1) eyes large in relation to body length $(17,3 \%)$, body height $(26,8 \%)$, and body width $(29,7 \%)$, interocular distance three times larger than eye diameter; (2) lower beak with two transverse stripes, proximal half white and distal half black.

KEY WORDS. Hylidae, Scinax similis, Scinax ruber group, larval morphology, Southeastern Brazil
\end{abstract}

Scinax similis (Cochran, 1952), um hilídeo cuja localidade-tipo é Manguinhos (Rio de Janeiro, estado do Rio de Janeiro), ocorre em áreas abertas, baixadas, brejos e restingas do sudeste do Brasil. Essa espécie tem sido incluída no grupo "ruber" (FouQueTtE \& Delahoussaye 1977; Duellman \& WiENS 1992, 1993), apresentando como as demais espécies de seu grupo uma taxonomia complexa.

A larva de $S$. similis, apesar de citada na literatura (CocHRAN 1952, 1955), não foi descrita formalmente, sendo difíceis a sua identificação e diferenciação em relação às larvas de outras espécies do grupo. CoCHRAN (1952), quando descreveu S. similis, teceu um breve comentário sobre a fragilidade e a rápida metamorfose de sua larva.

No presente trabalho descreve-se a larva de $S$. similis, comparando-a com larvas de Scinax hayii (Barbour, 1909) e Scinax fuscovarius (A. Lutz, 1925), espécies do grupo "ruber" que ocorrem no sudeste do Brasil. São também apresentadas algumas considerações sobre os hábitos da larva S. similis.

\section{MATERIAL E MÉTODOS}

As larvas de $S$. similis foram coletadas ao longo dos anos de 1992 e 1993, no Campus da Universidade Federal do Rio de Janeiro, Ilha do Fundão, estado do Rio de Janeiro, sendo encontradas em pequenas poças temporárias e, em sua maior

1) Departamento de Zoologia, Instituto de Biologia, Universidade Federal do Rio de Janeiro. Caixa Postal 68044, 21944-970 Rio de Janeiro, Rio de Janeiro, Brasil.

2) Museu Nacional. Quinta da Boa Vista, São Cristóvão, 20940-040 Rio de Janeiro, Rio de Janeiro, Brasil. 
parte, em tanques mantidos para criação de peixes. Esses tanques tinham praticamente toda sua superfície coberta por vegetação aquática (Salvinia sp.) e localizavam-se em área ensolarada ou parcialmente sombreada. Não foram obtidas desovas. As larvas e recém-metamorfoseados foram anestesiados em cloretona a $0,1 \%$ e fixados em solução de formaldeído a $0,5 \%$.

A descrição foi realizada com base em larvas no estágio 37 (GOSNER 1960). A determinação da fórmula dentária segue Altig (1970). As mensurações e nomenclatura de estruturas larvárias seguem parcialmente ALTIG (1970), exceto pela distância interocular, onde foi considerada a distância entre as margens mais internas dos globos oculares e pelos termos mandíbula e nadadeira, aqui tratados respectivamente por bico córneo e membrana. Para as medições foram utilizados paquímetro com precisão de $0,1 \mathrm{~mm}$ e ocular milimetrada em microscópio estereoscópio simples. Com o paquímetro foram medidos o comprimento total e comprimento do corpo, as demais medidas foram obtidas com a ocular milimetrada.

O material examinado está depositado na Coleção de Anfíbios do Departamento de Zoologia, Instituto de Biologia, Universidade Federal do Rio de Janeiro (ZUFRJ).

Material examinado. Scinax similis - Brasil, Rio de Janeiro: Rio de Janeiro (Ilha do Fundão), (ZUFRJ 5901), III/1992; (ZUFRJ 5902), 14/IV/1992; (ZUFRJ 5903), 22/IV/1992; (ZUFRJ 5904), 05/IV/1992; (ZUFRJ 5966), 04/III/1993; (ZUFRJ 5969), 03/XII/1992; (ZUFRJ 5970), 06/XII/1993. Scinax hayii - Brasil, Rio de Janeiro: Teresópolis (Vale da Revolta), (ZUFRJ 4394), 07/II/1991; (ZUFRJ 4395), 01/I/1991; (ZUFRJ 4396), 03/III/1991; (ZUFRJ 5787), 5-8/II/1991; Angra dos Reis (Ilha Grande), (ZUFRJ 4834), 18-19/II/1992; São Paulo: Ubatuba, (ZUFRJ 5708), 14-17/XII/1992. Scinax fuscovarius -Brasil, Rio de Janeiro: Nova Friburgo (Macaé de Cima) (ZUFRJ 3314), 19/IV/1987; São Gonçalo, (ZUFRJ 4813), X/1992; São Paulo: Botucatu (Rio Alambari), (ZUFRJ 5971), 22/III/1988; Trevo Rondon-Castelinho, (ZUFRJ 5972), 14/III/1986; Fazenda do Lageado, (ZUFRJ 5973), 15/XII/1988.

\section{Scinax similis (Cochran, 1952)}

Figs 1-5, 8

Descrição. Larva. Corpo globoso, oval em vista dorsal; mais alto que largo, correspondendo a $34,6 \%$ do comprimento total. Focinho, em vista dorsal, trapezoidal ou arredondado. Distância do olho ao focinho igual a $27,8 \%$ do comprimento do corpo. Olhos laterais, grandes tanto em relação ao comprimento do corpo $(17,3 \%)$ quanto à altura $(26,8 \%)$ e à largura $(29,7 \%)$, distando entre si cerca de três vezes o seu diâmetro. Narinas dorsais, de forma oval, visíveis em vista lateral; distância internasal com cerca de duas vezes a distância da narina ao focinho e ocupando $49,2 \%$ da largura do corpo. Espiráculo sinistro, localizado pouco depois da metade do corpo, curto, completamente preso a parede do corpo e orientado posterodorsalmente. Abertura do espiráculo em fenda. Tubo anal curto, ligado à membrana inferior da cauda, com abertura para o lado direito. Cauda representando $65,4 \%$ do comprimento total. Musculatura da cauda moderadamente desenvolvida, afilando gradualmente até formar uma ponta. Membranas da cauda com aproxima- 
damente o mesmo tamanho, bem desenvolvidas e vascularizadas. Membrana superior da cauda surgindo um pouco depois da metade do corpo, com contorno ascendente até o final do primeiro terço do comprimento da cauda, onde atinge seu ponto mais alto, a partir daí descendente, terminando em ponta fina e arredondada. Disco oral ântero-ventral, representando $45 \%$ da largura do corpo; papilas marginais achatadas e alongadas em quase toda extensão, ausentes numa pequena faixa mediana no lábio superior; papilas submarginais robustas e arredondadas, dispostas lateralmente em uma fileira irregular e outras distribuídas mais internamente de forma desordenada. Fórmula dentária: 2(2)/3(1); terceira fileira de dentículos do lábio inferior um pouco mais curta do que as duas primeiras. Bico córneo com bordas finamente serrilhadas; bico córneo superior arqueado com processos laterais; bico córneo inferior em forma de "V" aberto com espinho queratinizado localizado póstero-lateralmente.
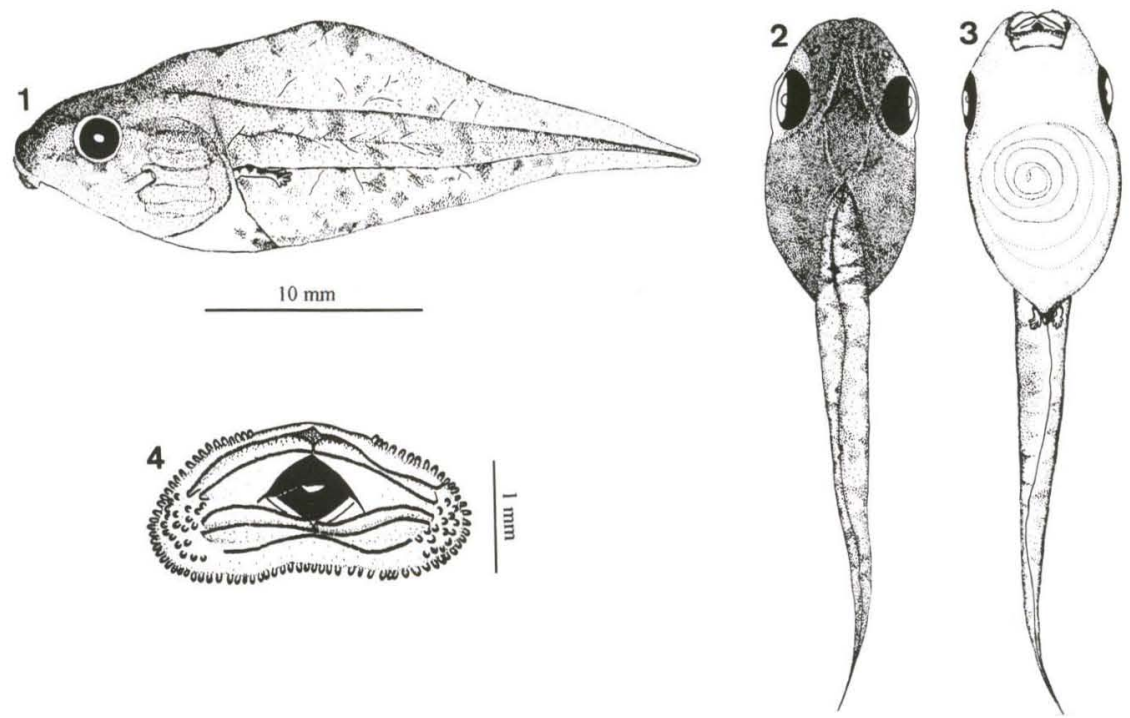

Figs 1-4. (1-4) Larva de Scinax similis (ZUFRJ 5904), estágio 37. (1) Vista lateral; (2) vista dorsal; (3) vista ventral; (4) disco oral.

Dimensões em milímetros de larvas no estágio $37(n=13)$ : $X \pm S d$ (mín.máx.). Comprimento total : $31,1 \pm 2,4(27,3-35,7)$; comprimento do corpo: $10,7 \pm 0,8$ $(9,0-12,0)$; altura do corpo: $7,0 \pm 0,7(5,7-8,3)$; largura do corpo: $6,3 \pm 0,5(5,2-7,1)$; comprimento da cauda: $20,4 \pm 1,8(17,6-23,7)$; altura da cauda: $10,3 \pm 1,0(8,4-12,0)$; altura da membrana superior da cauda: $3,6 \pm 0,4(3,0-4,4)$; altura da membrana inferior da cauda: $3,6 \pm 0,5(2,4-4,6)$; distância do focinho ao surgimento da cauda: 5,8 $\pm 0,4$ (4,4-6,3); distância da narina ao focinho: $1,4 \pm 0,2$ (1,0-1,9); distância internasal: $3,1 \pm 0,2(2,7-3,4)$; diâmetro da narina: $0,4 \pm 0,1(0,4-0,5)$; distância do olho ao focinho: $3,0 \pm 0,2(2,3-3,3)$; distância interocular: 5,6 $\pm 0,2(5,2-6,0)$; diâmetro do olho: $1,8 \pm 0,1(1,7-2,1)$; distância do olho à narina: $1,2 \pm 0,1(1,0-1,3)$; largura do disco oral: $2,8 \pm 0,3(2,3-3,3)$. 

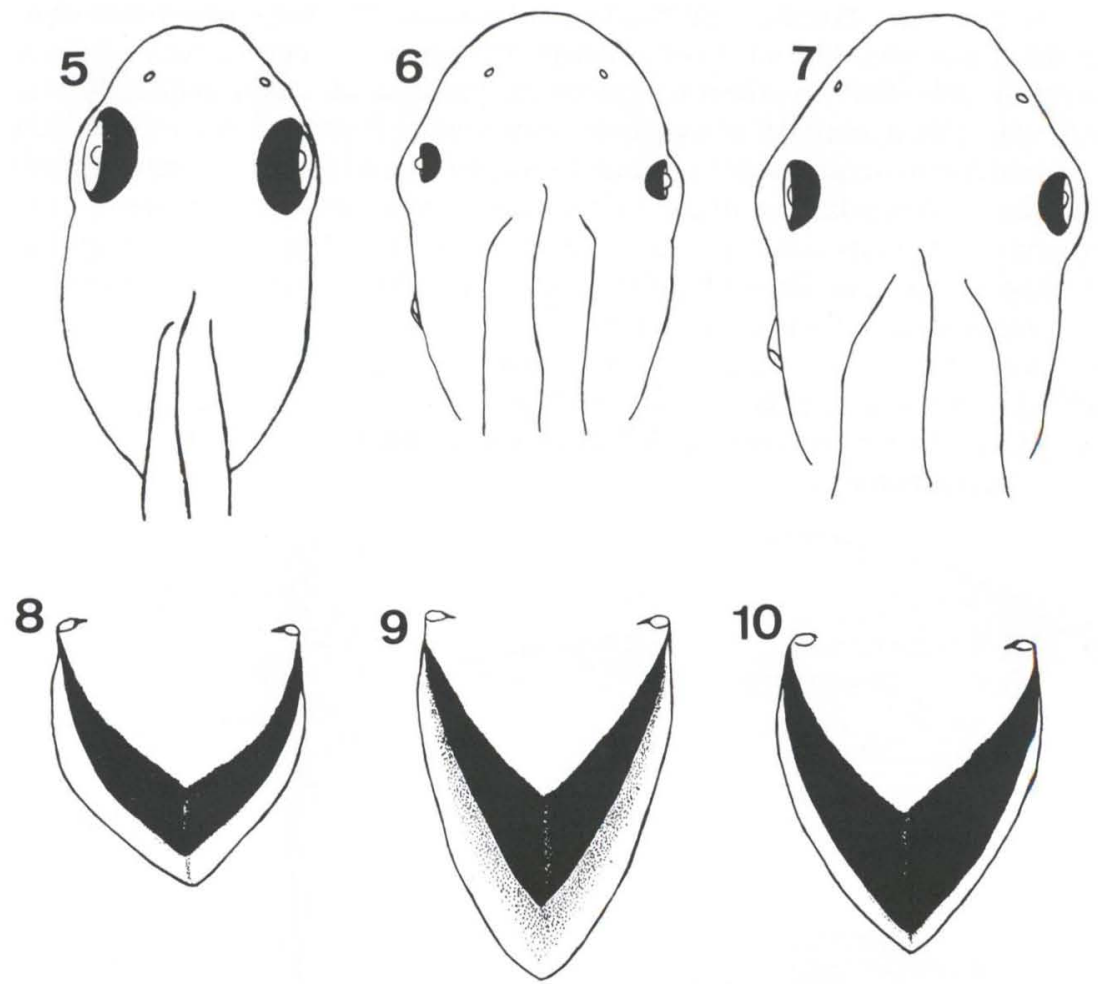

Figs 5-10. (5-7) Larvas, esquema em vista dorsal: (5) S. similis; (6) S. hayii, (7) S. fuscovarius. (8-10) Bico córneo inferior: (8) S. similis; (9) S. hayii; (10) S. fuscovarius.

Coloração em vida. Dorso castanho-amarelado e ventre acobreado. Faixa lateral, formada por pontuações castanhas, estendendo-se olho do até o focinho. Olhos dourados. Membranas da cauda transparentes, amareladas, com maior concentração de pigmento nos dois primeiros terços, quase incolores na porção terminal. Pontuação castanho escuro em todo corpo e cauda.

Coloração no fixador. Corpo e musculatura da cauda creme, levemente amarelados, com pontuações castanhas. Dorso com pontuação castanha muito concentrada, o que lhe confere uma aparência mais escura; ventre transparente, levemente esbranquiçado, com pontuações castanhas e brancas bem esparsas. Faixa lateral olho-focinho nem sempre nítida. Intestino cinza escuro, destacando-se lateralmente e ventralmente por transparência. Musculatura da cauda apresentando pontos castanhos mais concentrados nas regiões próximas às membranas, no centro e na porção final. Membranas transparentes, com manchas irregulares, formadas por pontuação castanha, mais concentradas nas bordas e perto da musculatura. Coloração geral do bico córneo escura; bico córneo inferior branco na metade proximal e preto na metade distal. 
Aspectos biológicos e ecológicos. As larvas de S. similis podem ser encontradas de dezembro até maio. São animais de ambientes lênticos e de hábitos nectônicos. Em cativeiro, costumam raspar a vegetação do fundo, as paredes dos aquários e a vegetação flutuante, ocupando toda massa d'água. Mantêm-se tanto na posição horizontal quanto na vertical (com a boca voltada para baixo ou para cima) e usualmente deslocam-se lentamente batendo apenas a porção terminal da cauda. A densidade de pigmentação das larvas é variável com o ambiente, sendo freqüentemente mais intensa e brilhante no ambiente onde foram coletadas.

O comprimento rostro-anal, em milímetros, dos jovens recém-metamorfoseados $(\mathrm{n}=21)$ é de $12,4 \pm 1,3(11-15,5)$. Os indivíduos mantidos no laboratório até a metamorfose apresentaram tamanho maior do que aqueles coletados no ambiente.

Tabela I. Proporções, em porcentagem, de Scinax similis, S. hayii e S. fuscovarius no estágio 37 [média \pm desvio padrão (máximo-mínimo)]. (CTOT) Comprimento total, (CCOR) comprimento do corpó, (ACOR) altura do corpo, (LCOR) largura do corpo, (CCAU) comprimento da cauda, (ACAU) altura da cauda, (DFSC) distância do focinho ao surgimento da cauda, (DIN) distância internasal, (DOLH) diâmetro do olho, (DO) disco oral.

\begin{tabular}{|c|c|c|c|c|c|c|c|c|c|c|c|c|}
\hline \multirow[b]{2}{*}{ CCOR/CTOT } & \multicolumn{4}{|c|}{ Scinax similis $(n=13)$} & \multicolumn{4}{|c|}{ Scinax hayii $(n=10)$} & \multicolumn{4}{|c|}{ Scinax fuscovarius $(n=10)$} \\
\hline & 34,6 & 1,7 & $(31,2$ & $-38,1)$ & 37,2 & 1,5 & $(35,6$ & $-40,4)$ & 32,0 & \pm & 1,7 & $(27,8-34,4)$ \\
\hline ACOR/CCOR & 64,8 & 3,4 & $(58,2$ & $-71,6)$ & 66,2 & 3,7 & $(59,5$ & $-72,8)$ & 66,1 & \pm & 3,0 & $(60,2-71,4)$ \\
\hline LCOR/CCOR & 58,4 & 2,0 & $(53,8$ & $-61,7)$ & 60,5 & 2,4 & $(57,4$ & $-65,3)$ & 56,8 & \pm & 3,0 & $(52,3-64,4)$ \\
\hline DFSCICCOR & 53,8 & 3,3 & $(47,5$ & $-59,0)$ & 47,1 & 2,7 & $(41,6$ & $-51,6)$ & 45,1 & \pm & 1,7 & $(43,5-49,6)$ \\
\hline DOLH/ACOR & 26,8 & 2,0 & $(23,1$ & $-29,8)$ & 17,7 & 1,1 & $(15,8$ & -19 & 23,6 & \pm & 1,9 & $(20,7-26,9)$ \\
\hline DOLH/LCOR & 29,7 & 2,1 & $(27,0$ & $-33,3)$ & 19,3 & 0,8 & $(17,9$ & $-20,6)$ & 27,5 & \pm & 2,2 & $(22,9-31,4)$ \\
\hline DOLH/CCOR & 17,3 & 0,9 & $(16,0$ & $-19,0)$ & 11,7 & 0,8 & $(10,3$ & $-13,0)$ & 15,6 & \pm & 0,9 & $(14,4-17,9)$ \\
\hline ACAU/CCAU & 50,6 & 4,3 & $(44,4$ & - 60,4) & 55,1 & 3,8 & $(48,8$ & $-63,3)$ & 42,0 & \pm & 2,5 & $(37,9-45,9)$ \\
\hline DIN/LCOR & 49,2 & 1,8 & $(46,5$ & $-52,6)$ & 48,8 & 1,8 & $(45,9$ & $-51,5)$ & 57,3 & \pm & 3,7 & $(52,5-63,6)$ \\
\hline DO/LCOR & 45,0 & 3,7 & $(40,4$ & $-52,6)$ & 50,5 & 1,2 & $(47,9$ & $-52,3)$ & 42,2 & \pm & 1,9 & $(39.5-44,8)$ \\
\hline
\end{tabular}

\section{DISCUSSÃO E CONCLUSÕES}

As larvas de $S$. similis, $S$. hayii e $S$. fuscovarius são muito semelhantes, o que torna difícil a identificação das mesmas. O tamanho dos olhos (Tab. I, Figs 5-7) em relação ao comprimento, altura e largura do corpo e a coloração do bico córneo inferior (Figs 8-10) mostraram-se como os caracteres diferenciais mais úteis. Em relação ao bico córneo inferior, observou-se a presença de um espinho queratinizado, localizado látero-posteriormente nas três espécies. A larva de $S$. similis possui olhos grandes tanto em relação ao comprimento do corpo $(17,3 \%)$, quanto em relação à altura $(26,8 \%)$ e à largura $(29,7 \%)$, distando entre si cerca de três vezes o seu diâmetro; coloração do bico córneo inferior branca na metade proximal e preta na distal; coloração em vida amarelada. As larvas de $S$. hayii analisadas nesse estudo enquadram-se nas descrições de BOKERMANN (1967) e HEYER et al. (1990), distinguindo-se de $S$. similis por uma aparência mais globosa; relações entre o diâmetro dos olhos e o comprimento, altura e largura do corpo menores $(11,7 \%$; $17,7 \%$ e $19,3 \%$ respectivamente) do que em S. similis; distância interocular cerca de cinco vezes o diâmetro do olho; bico córneo inferior branco na faixa proximal, marrom na mediana e preto na distal; coloração em vida olivácea. As larvas de $S$. fuscovarius enquadram-se na descrição de VizotTo (1967), distinguindo-se de $S$.

Revta bras. Zool. 16 (2): 507 - 512, 1999 
similis por relações entre o diâmetro dos olhos e o comprimento, altura e largura do corpo também um pouco menores $(15,6 \% ; 23,6 \%$ e $27,5 \%$ respectivamente); distância interocular cerca de quatro vezes o diâmetro do olho; bico córneo inferior predominantemente preto; coloração em vida olivácea.

AGRADECIMENTOS. O manuscrito foi cuidadosamente revisado pelos seguintes pesquisadores: Márcia R. Gomes, Eugênio Izecksohn, Gabriel Mejdalani, Oswaldo L. Peixoto, Richard Sachsse e Elieth F. Spirandeli Cruz. Jorge Jim e Elieth F. Spirandeli Cruz doaram o material de Scinax fuscovarius. Esse estudo foi desenvolvido com apoio financeiro do CNPq.

\section{REFERÊNCIAS BIBLIOGRÁFICAS}

ALTIG, R. 1970. A key to the tadpoles of the continental United States and Canada. Herpetologica 26: 180-207.

BOKERMANN, W.C.A. 1967. Girinos de anfíbios brasileiros - 4 (Amphibia, Salientia). Rev. Brasil. Biol. 27 (4): 363-367.

Cochran, D.M. 1952. Two Brazilian frogs: Hyla werneri n.nov., Hyla similis n.sp. Jour. Wash. Acad. Sci. 42 (2): 50-53.

- 1955. Frogs of southeastern Brazil. Bull. U.S. Nat. Mus. 206: 1-423.

Duellman, W.E. \& J.J. WiENS. 1992. The status of the Hylid frog genus Ololygon and the recognition of Scinax Wagler, 1830. Univ. Kansas Mus. Nat. Hist. Occ. Pap. 151: 1-23.

- 1993. Hylid frogs of the genus Scinax Wagler, 1830, in Amazonian Ecuador and Peru. Univ. Kansas Mus. Nat. Hist. Occ. Pap. 153: 1-57.

FouQueTte JR., M.J. \& A.J. DelahousSAYe. 1977. Sperm morphology in the Hyla rubra group (Amphibia: Anura: Hylidae), and its bearing on generic status. Jour. Herpetol. 11 (4): 387-396.

GosNER, K.L. 1960. A simplified table for staging anuran embryos and larvae with notes on indentification. Herpetologica 16: 183-190.

Heyer, W.R.; A.S. Rand; C.A.G. CruZ; O.L. PeiXoto \& C.E. Nelson. 1990. Frogs of Boracéia. Arq. Zool., São Paulo, 31 (4): 231-410.

VizotTo, L.D. 1967. Desenvolvimento de anuros da região norte-ocidental do Estado de São Paulo. Faculdade de Filosofia, Ciências e Letras de São José do Rio Preto, Zoologia (Número especial): 1-161.

Recebido em 11.XII.1997; aceito em 13.IV.1999. 